\title{
Analysis of Output Loading Effects in Autonomous Circuits
}

\author{
Almudena Suárez, Fellow, IEEE, Mabel Pontón, Member, IEEE, Sergio Sancho, Member, IEEE, and \\ Franco Ramírez, Senior Member, IEEE
}

\begin{abstract}
A methodology is presented to analyze the impact of the termination load on the oscillation frequency and output power of autonomous circuits. Variations of this load can also lead to an extinction of the oscillation signal, due to their effect on the impedance seen by the active device(s). The new methodology enables an efficient analysis and mitigation of the pulling effects, in the case of undesired output mismatch, as well as an efficient oscillator synthesis in large-signal conditions, for specified values of oscillation frequency and output power. The method is based on the calculation of constant-amplitude and constant-frequency contours, traced in the Smith chart. Oscillation extinctions and some forms of hysteresis can be predicted through the inspection of these contours. However, the stability properties will generally depend on the frequency characteristic of the termination impedance. In an oscillator synthesis, the selected impedance, providing the specified values of oscillation frequency and output power, must be implemented in order to guarantee a stable solution. The dependence of the phase-noise spectral density on the particular implementation is predicted combining an analysis based on the variance of the phase deviation with the conversionmatrix approach.
\end{abstract}

Index Terms- Antenna mismatch, stability analysis, bifurcation analysis, frequency pulling, phase noise.

\section{INTRODUCTION}

$M$ OST nonlinear circuits are designed under the assumption of an ideal $50 \Omega$ termination load, though, in practice, the output load may be different from this standard value, which is a common situation when the nonlinear circuit is connected to an antenna [1]-[3]. The mismatched conditions may be due to an antenna circuit with input impedance different from $50 \Omega$ or be the result of reflections in objects in the neighborhood [1]-[7]. In power amplifiers (PAs), the mismatch effects can give rise to significant degradation in output power, efficiency and linearity [1]-[3]. However, the most disruptive effect is the possible circuit instability [6]-[7].

Manuscript submitted January 15, 2017. This paper is an expanded version from the IEEE MTT-S Latin America Microwave Conference, Dec. 12-14, 2016, Puerto Vallarta, Mexico. This work was supported by the Spanish Ministry of Economy and Competitiveness under the research project TEC2014-60283-C3-1-R, the European Regional Development Fund (ERDF/FEDER) and Juan de la Cierva Research Program IJCI-2014-19141 and by the Parliament of Cantabria under the project Cantabria Explora 12.JP02.64069.

A. Suárez, M. Pontón, S. Sancho, and F. Ramírez are with Dpto. Ingeniería de Comunicaciones, Universidad de Cantabria. Av. Los Castros 39005 Santander. Spain (e-mail: suareza@unican.es; pontonmi@unican.es; ramirezf@unican.es; sanchosm@unican.es).
Unless a specific design criterion is used, PA manufacturers cannot guarantee stable behavior for all the possible termination impedances. In view of this need, in [8]-[11], a potential instability analysis is addressed, taking into account that the PA termination impedances are unknown, unlike the case of standard stability analyses [12]-[14], in which all the circuit elements and parameters are specified. It is a largesignal stability analysis, so the PA input frequency $f_{\text {in }}$, as well as an incommensurable perturbation frequency $f$ must be considered. As a result, the analysis must be carried out in the presence of harmonic and sideband frequencies. However, the PA output network will exhibit filtering effects [8]-[11], so only the termination impedances at $f_{\text {in }}$ and two or three sideband frequencies (depending on the filtering characteristic) will have an impact on the stability properties. The potential instability analysis in [8]-[11] is based on the definition of a low-order outer-tier scattering matrix at the relevant sideband frequencies, calculated with the conversion-matrix approach. This enables the evaluation of several large-signal equivalents of the $\mu$ factor [15]. For unconditional stability, these factors should be larger than one for all passive load impendences at $f_{\text {in }}$ and all perturbation frequencies in the interval $\left(0, f_{\text {in }}\right)$.

Performance degradation due to output mismatch can also be expected in oscillators connected to an antenna or based on active antennas, when operating in reflective environments [3]. Among the possible undesired effects is the frequency pulling, or oscillation-frequency variations due to reflections from the load [16]-[19]. Some previous works on frequency pulling focus on the experimental characterization of this phenomenon, such as [18]-[19], or propose a linear open-loop simulation [20] of the oscillator transmission amplitude and phase, under variations of the load reflection coefficient. Analytical studies, such as those in [16], [21]-[22], are very insightful, but assume a small variation of the output load, so that the oscillator total admittance or impedance function can be linearized with respect to this load.

This paper extends the work in [8] with a detailed investigation of mismatch effects in autonomous circuits. Unlike previous works, the new simulation method considers all possible variations of the passive output load, covering the entire Smith chart. It is able to predict the possible oscillation extinction for some loading conditions, which would lead the circuit to an undesired dc regime. This phenomenon has not been analyzed in any previous work to our knowledge.

The new analysis method relies on the calculation through harmonic balance (HB) of the circuit output-admittance function under different amplitude and frequency excitations. 
By means of this function, constant amplitude and frequency contours are traced in the output-load Smith chart. This is done without any specific oscillator-analysis procedures, such as the ones based on the use of "osc-ports" or probes [23]-[24]. The method can be applied for an efficient oscillator synthesis, since the constant-amplitude and constant-frequency contours enable a straight-forward selection of the load impedance for specified values of the oscillation frequency and output power. This may have a particular interest for the design of reconfigurable oscillators, in which the oscillation frequency changes through a modification of the loading conditions [25]-[26].

Some forms of hysteresis [24],[27]-[28], as well as the oscillation extinction, are detectable with a simple inspection of the geometry of the constant-amplitude or frequency contours. The onset of additional incommensurable or subharmonic oscillations is also possible, as in the forced case. However, in most cases, the stability properties [12]-[14] will depend on the frequency characteristic of the output load. When using the method for oscillator synthesis, different implementations of a selected impedance value will provide either stable or unstable behavior, which can be predicted through pole-zero identification [12]-[14]. On the other hand, internal instabilities are easily detected by considering the limit cases of open and short circuits at the sideband frequencies [11]. As expected, the phase-noise spectrum [29]-[30] will depend on the particular implementation of the output load, which will be analyzed with a method based on the calculation of the phase variance [31], in combination with the conversion-matrix approach [32]-[33].

The paper is organized as follows. To emphasize the differences between the forced and autonomous cases, Section II presents a brief summary of the method to predict potential instabilities in PAs under mismatch effects. The remainder of the paper is devoted to the autonomous case. Section III provides a methodology for an efficient analysis of the steadystate oscillation versus variations in the output load, with a direct application in oscillator synthesis. Section IV presents the main instability mechanisms. Section V studies the effect of the oscillator load on the phase-noise spectral density.

\section{MisMATCHED-INDUCED INSTABILITY OF PAS}

In a power amplifier, the stability analysis under mismatch effects is carried out at the output reference plane [Fig. 1(a)], where it is connected to the antenna [8]-[11]. The PA will behave nonlinearly with respect to the input source, this resulting in a certain spectrum at $k f_{i n}$, where $k$ is an integer. To analyze the possible instability under mismatch effects, a small perturbation at the frequency $f[6],[8]-[11]$ must also be considered. In fact, the stability analysis is a prediction of the circuit reaction to small perturbations coming from noise or fluctuations. The perturbation frequency gives rise to the mixing terms $k f_{\text {in }}+f[6],[8]-[11]$. Thus, the analysis must take into account the harmonic frequencies $k f_{i n}$, as well as the sideband frequencies $k f_{\text {in }}+f$, such that $-N H<k<N H$, where $N H$ is the number of harmonic terms. Reactive impedances necessarily exhibit frequency dependence, so the termination load $Z_{L}(f)$ will be different at each harmonic and sideband frequency, and all possible combinations of passive impedance values should be considered. The impedance terminations at $k f_{\text {in }}$ will have an impact on the large-signal periodic solution and those at $k f_{i n}+f$ will have an impact on the circuit linearization with the conversion-matrix approach. In most cases, and due to the filtering effects of the output network, the only sensitive impedance terminations will correspond to $f_{i n}$, $f_{b}=f, f_{l}^{*}=-f_{\text {in }}+f$ and $f_{u}=f_{\text {in }}+f$, so the rest of frequency terminations can be arbitrarily terminated in $Z_{L}=50 \Omega$.

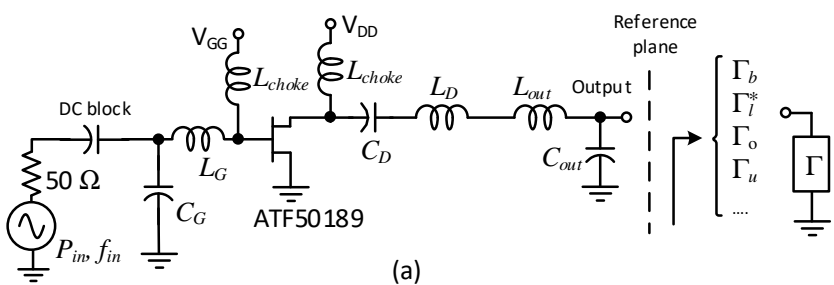

(a)

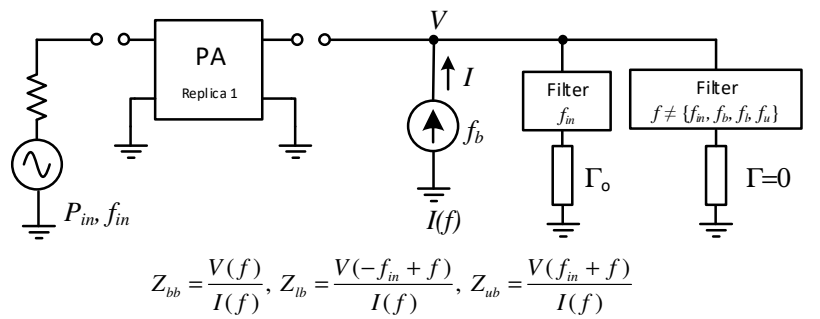

(b)

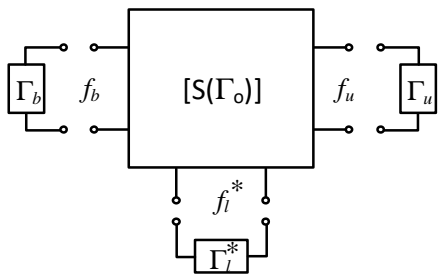

(c)

Fig. 1. Potential instability analysis of PAs under output-mismatch effects. (a) Schematic of application example, indicating the analysis reference plane. The transistor is an Avago ATF-50189, and the element values are $L_{\text {choke }}=$ $150 \mathrm{nH}, C_{D}=2.2 \mathrm{pF}, L_{D}=18 \mathrm{nH}, L_{\text {out }}=8 \mathrm{nH}, C_{\text {out }}=1.5 \mathrm{pF}, L_{G}=2.5 \mathrm{nH}$ and $C_{G}=1.5 \mathrm{pF}$. The PA operation frequency is $f_{\text {in }}=0.8 \mathrm{GHz}$ and the nominal input power is $P_{\text {in }}=11.93 \mathrm{dBm}$. (b) Illustration of the current excitation at $f_{b}$ to calculate the first column of the impedance matrix $\left[\mathrm{Z}_{3}\right]$ through the ratios in (1.a). (c) Outer-tier Scattering matrix describing the mismatched PA response at the three relevant sideband frequencies $f_{b}, f_{l}^{*}$ and $f_{u}$.

The potential instability analysis, which must considered all possible terminations $\Gamma_{o}$ at $f_{i n}$, is carried out as follows. The steady-state solution when terminating the circuit with $\Gamma_{o}$ is calculated through $\mathrm{HB}$. Then, a $3 \times 3$ impedance matrix $\left[\mathrm{Z}_{3}\right]$ is obtained [8]-[11] considering 3 virtual ports at $f_{b}, f_{l}^{*}$ and $f_{u}$ [Fig. 1(b) and Fig. 1(c)]. The rest of frequencies, arbitrarily terminated in $\Gamma=0$, are taken into account in an inner tier. The components of $\left[\mathrm{Z}_{3}\right]$ are obtained by sequentially exciting the circuit with a small-signal current source at $f_{b}, f_{l}^{*}$ and $f_{u}$, by means of the conversion-matrix approach [32]-[33]. See Fig. 1(b) for an illustration of the current excitation at $f_{b}=f$, with the circuit terminated in $\Gamma_{o}$ at $f_{i n}$, in open circuit at $f_{b}, f_{l}^{*}$ and $f_{u}$ and in $\Gamma=0$ at the rest of harmonics and sidebands. Analogous arrangements are used for the current excitations at $f_{l}^{*}$ and $f_{u}$. 
Then, the elements of the outer-tier matrix $\left[\mathrm{Z}_{3}\right]$ are calculated with the following voltage-to-current ratios.

$$
\begin{gathered}
Z_{b b}=\frac{V(f)}{I(f)}, Z_{l b}=\frac{V\left(-f_{i n}+f\right)}{I(f)}, Z_{u b}=\frac{V\left(f_{i n}+f\right)}{I(f)} \\
Z_{b l}=\frac{V(f)}{I\left(-f_{i n}+f\right)}, Z_{l l}=\frac{V\left(-f_{\text {in }}+f\right)}{I\left(-f_{\text {in }}+f\right)}, Z_{u l}=\frac{V\left(f_{\text {in }}+f\right)}{I\left(-f_{\text {in }}+f\right)} \\
Z_{b u}=\frac{V(f)}{I\left(f_{\text {in }}+f\right)}, Z_{l u}=\frac{V\left(-f_{\text {in }}+f\right)}{I\left(f_{\text {in }}+f\right)}, Z_{u u}=\frac{V\left(f_{\text {in }}+f\right)}{I\left(f_{\text {in }}+f\right)}
\end{gathered}
$$

To be more specific, the first column of $\left[Z_{3}\right]$ is calculated with the excitation current source at $f_{b}$, as shown in (1.a). The second column is calculated with the current source at $f_{l}^{*}$, as shown in (1.b). The third column of $\left[Z_{3}\right]$ is calculated with the currents source at $f_{u}$, as shown in (1.c). The matrix $\left[\mathrm{Z}_{3}\right]$ depends on the perturbation frequency, so the current-source frequency $f$ must be swept through the entire interval $\left(0, f_{\text {in }}\right)$ in the three cases.

The matrix $\left[Z_{3}\right]$ (which is different for each termination $\Gamma_{o}$ ) is transformed into a scattering matrix $\left[\mathrm{S}_{3}\right]$, with three virtual ports at the three relevant sidebands $f_{b}, f_{l}{ }^{*}$ and $f_{u}$ [Fig. 1(c)]. This matrix can be reduced to a $2 \times 2$ one when considering a particular termination $\Gamma_{s b}$ at any of the three sidebands. This way it is possible to obtain 3 different large-signal equivalents $\mu_{b, u}, \mu_{b, l}$ and $\mu_{l, u}$ of the stability factor [15]. Each factor corresponds to the $2 \times 2$ matrix defined between two of the sidebands $f_{b}, f_{l}^{*}$ and $f_{u}$, with the third sideband terminated in $\Gamma_{s b}$. For unconditional stability, the three factors must be larger than one for all values of $\Gamma_{o}$ and $f$ in $\left(0, f_{i n}\right)$. In ordinary cases, the most critical stability situation will correspond to $\Gamma_{s b}=1 e^{j \phi}$ [11]. Under sufficient observability, when sweeping $\phi$ from $0^{\circ}$ to $360^{\circ}$, and taking the minima of the three large-signal $\mu$ factors, the same prediction on the circuit potential instability will be obtained. Due to the nonlinearity of the problem, the factors depend on the input power $P_{i n}$, besides $\Gamma_{o}$ and $f$. The instability due to output mismatch effects should be detectable from the output-reference plane. The possible internal origin of the instability problem must be verified through a generalization of the Rollet proviso [34]. According to this proviso [11], for each termination $\Gamma_{o}$ at $f_{i n}$, the PA must be stable under all possible combinations of open- and shortcircuit terminations at the three sidebands $f_{b}, f_{l}^{*}$ and $f_{u}$. This can be verified with pole-zero identification [12]-[14].

The method has been applied to a PA based on the HEMT Avago ATF-50189, operating at $f_{i n}=0.8 \mathrm{GHz}$ [Fig. 1(a)]. The PA exhibits $80 \%$ drain efficiency at the output power $P_{\text {out }}=22$ $\mathrm{dBm}$, with the input power $P_{\text {in }} \cong 12 \mathrm{dBm}$. The output inductor and equivalent load resistance are $L_{\text {out }}=8 \mathrm{nH}$ and $R=41.5 \Omega$. The resistance $R$ is implemented through an L-C matching section, terminated in $50 \mathrm{Ohms}$. All circuit element values are provided in the caption of Fig. 1. Fig. 2 presents the frequency-variation of the phase-minima of the three factors of the PA in Fig. 1(a), for $P_{\text {in }}=11.93 \mathrm{dBm}$, and fundamentalfrequency termination $\Gamma_{o}=0.557 e^{j 150^{\circ}}$. For absolute stability the factors must be larger than 1 for every perturbation frequency $f$. This should be fulfilled for each fundamentalfrequency termination $\Gamma_{o}$.

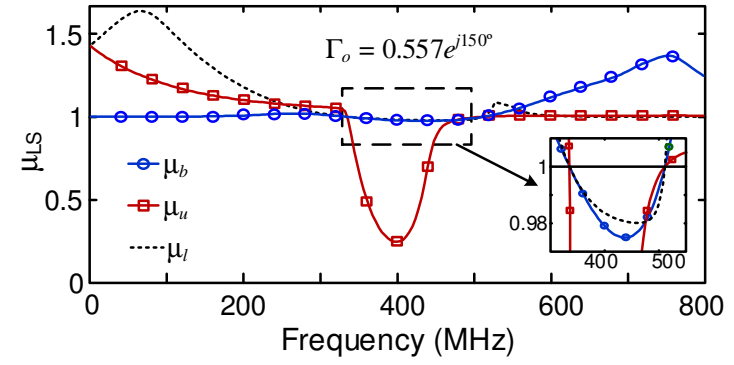

Fig. 2. Variation of the minima of the three stability factors $\mu_{b, u}(\phi), \mu_{b, l}(\phi)$ and $\mu_{l, u}(\phi)$, with $\left|\Gamma_{s b}\right|=1$, versus the perturbation frequency $f$, for $P_{\text {in }}=11.93$ $\mathrm{dBm}$ and the particular fundamental-frequency termination $\Gamma_{o}=0.557 e^{j 150^{\circ}}$.

To determine the terminations $\Gamma_{o}$ giving rise to potential instability one should trace the contour plots $\mu_{L S}^{\min }\left(\Gamma_{o}\right)=\min _{f}\left[\mu_{L S}\left(\Gamma_{o}, \Gamma_{s b}, f\right)\right]=1$, where $\mu_{L S}$ refers to any of the three large-signal factors [11], on the $\Gamma_{o}$ Smith chart. Note that the global coefficient $\mu_{L S}^{\min }\left(\Gamma_{o}\right)$ corresponds to the minimum value of the large-signal stability factor with respect to both $\Gamma_{s b}$ and $f$. The results of this analysis are shown in Fig. 3(a). They indicate potential instability since a significant region of the Smith chart exhibits $\mu_{L S}^{\min }\left(\Gamma_{o}\right)<1$. The results have been experimentally validated by loading the circuit with a triple-stub tuner. Fig. 3(b) shows the measured impedance plots for which stable behavior and undesired oscillations have been experimentally obtained, represented with squares and circles, respectively. They are consistent with the predictions of potentially unstable behavior in Fig. 3(a). The (overlapped) experimental spectra corresponding to the stable and unstable loads are shown in Fig. 3(c) and 3(d), respectively. The spectra in Fig. 3(d) contain intermodulation products with an undesired oscillation frequency.

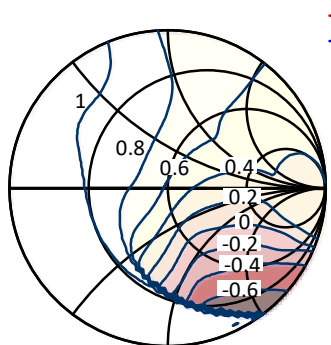

(a)

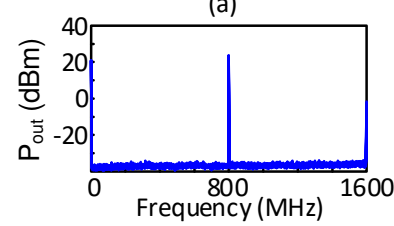

(c)

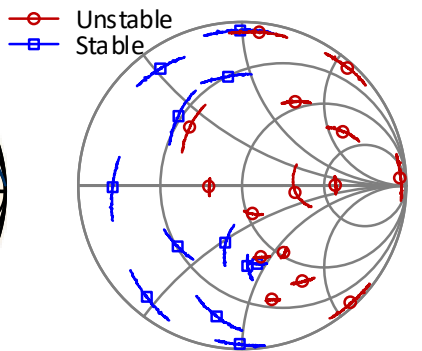

(b)

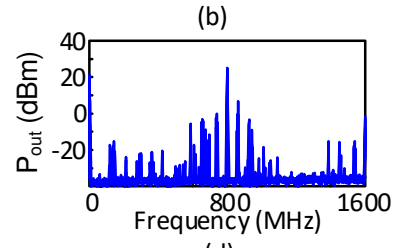

(d)
Fig. 3. Contour plots of $\mu_{L S}^{\min }\left(\Gamma_{o}\right)$. (a) Simulations, indicating potential instability since a significant region of the Smith chart exhibits $\mu_{L S}^{\min }\left(\Gamma_{o}\right)<1$. (b) Experimental validation with a triple stub tuner. (c) Ensemble of output spectra for plots marked with squares. (d) Ensemble of spectra for plots marked with circles, exhibiting intermodulation products with an undesired oscillation frequency. 


\section{VARIATION OF THE OUTPUT LOAD IN AN OSCILLATOR CIRCUIT}

The case of an oscillator circuit will be considered in this section. The impact of the output load on the oscillation frequency and amplitude will be analyzed with an efficient HB method. The applicability of this method requires sufficient filtering effects in the oscillator output network, so as to ensure that only the termination load at the fundamental frequency $f_{o}$ is relevant to the circuit solution. The method will be illustrated through its application to the oscillator circuit in Fig. 4, based on the transistor NE3210S01. A second harmonic trap (parallel L-C network) is introduced in the output network, prior to the termination load, and the effect of this load at higher harmonic terms, much lower than the fundamental, is neglected. Note that more selective filter configurations may be necessary in other cases.
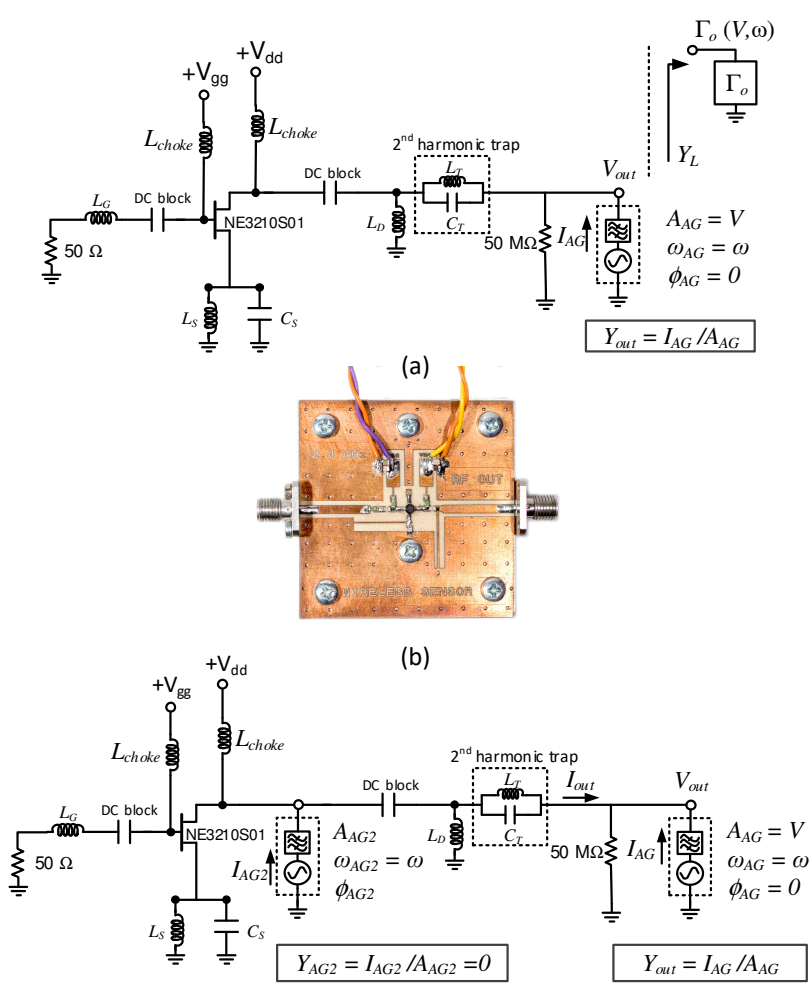

(c)

Fig. 4. Analysis of the impact of the output load on the steady-state solution of an oscillator circuit. (a) Schematic of the oscillator. The auxiliary generator AG1 is used to obtain the current-to-voltage ratio $Y_{\text {out }}(V, \omega)$ under different amplitude and frequency excitations. (b) Experimental prototype built on Rogers 4003C substrate. (c) Setup for the calculation of multivalued functions $Y_{\text {out }}(V, \omega)$. It is based on the use of two auxiliary generators, AG1 (for excitation) and AG2 (for oscillator analysis).

The HB analysis is carried out with an arbitrary number of harmonic terms $N H$. However, due to the presence of the output filter, it is assumed that only the termination impedance at the fundamental frequency $\omega_{b}=2 \pi f_{o}$, which is denoted as $\Gamma_{o}$, will have an impact on the oscillation amplitude and frequency. The analysis is carried out by opening the oscillator circuit at the termination-output node [Fig. 4(a)]. No load is connected to this node, though the introduction of a high value resistor (such as $R=50 \mathrm{M} \Omega$ ) is advisable to avoid any $\mathrm{HB}$ convergence problems. Then, an auxiliary generator (AG1) is connected in parallel, between the output node and ground. It is a voltage generator with amplitude $A_{A G}=V$, frequency $\omega_{A G}=\omega$ and phase arbitrarily set to zero, $\phi_{A G}=0$, with an ideal bandpass filter at $\omega_{A G}$ [Fig. 4(a)]. Unlike previous works [28], [35], this generator will not fulfill a non-perturbation condition, since its aim is to calculate the current-to-voltage ratio $Y_{\text {out }}(V, \omega)$ when looking into the circuit output under different excitation amplitudes $V$ and frequencies $\omega$. This function is given by:

$$
Y_{\text {out }}(V, \omega)=\frac{I_{A G}}{A_{A G}}
$$

where $I_{A G}$ is the current circulating through the AG at $\omega$ (and entering the circuit). The function (4) is calculated with $\mathrm{NH}$ harmonic terms, since these terms may be significant at internal circuit nodes. Applying the steady-state oscillation condition at the output node [36], an oscillation of amplitude $V$ and frequency $\omega$ should be achieved when loading the circuit with the admittance:

$$
Y_{L}(\omega)=-Y_{\text {out }}(V, \omega)
$$

where the subindex " $o$ " in the fundamental frequency has been dropped for notation simplicity. Remember that at the output node, the impact of the load admittance values at harmonic terms $|N H|>1$ has been considered negligible, due to the output filter. The load reflection coefficient at the fundamental frequency is directly calculated from (5), as:

$$
\Gamma_{o}(V, \omega)=\frac{Y_{c}-Y_{L}}{Y_{c}+Y_{L}}
$$

where $Y_{c}=1 / 50 \Omega^{-1}$. The voltage $V$ and frequency $\omega$ inside the parentheses indicate the values of the oscillation amplitude and frequency obtained when terminating the oscillator circuit in the reflection coefficient $\Gamma_{o}$.

The load values $Y_{L}(\omega)$ fulfilling (5) are calculated in a systematic manner by performing a double sweep in the amplitude $A_{A G}=V$ and frequency $\omega_{A G}=\omega$ of the auxiliary generator [AG1 in Fig. 4(a)]. An outer sweep in $A_{A G}$ with a finer sweep in $\omega_{A G}$ enables the calculation of constantamplitude contours, of the form $\Gamma_{o}\left(V_{o}, \omega\right)$, where $V_{o}$ is a specific amplitude value. In turn, an outer sweep in $\omega_{A G}$ with a finer sweep in $A_{A G}=V$ enables the calculation of constantfrequency contours $\Gamma_{o}\left(V, \omega_{o}\right)$. For the reader's convenience the different stages of the analysis procedure are indicated in the flowchart of Fig. 5. 


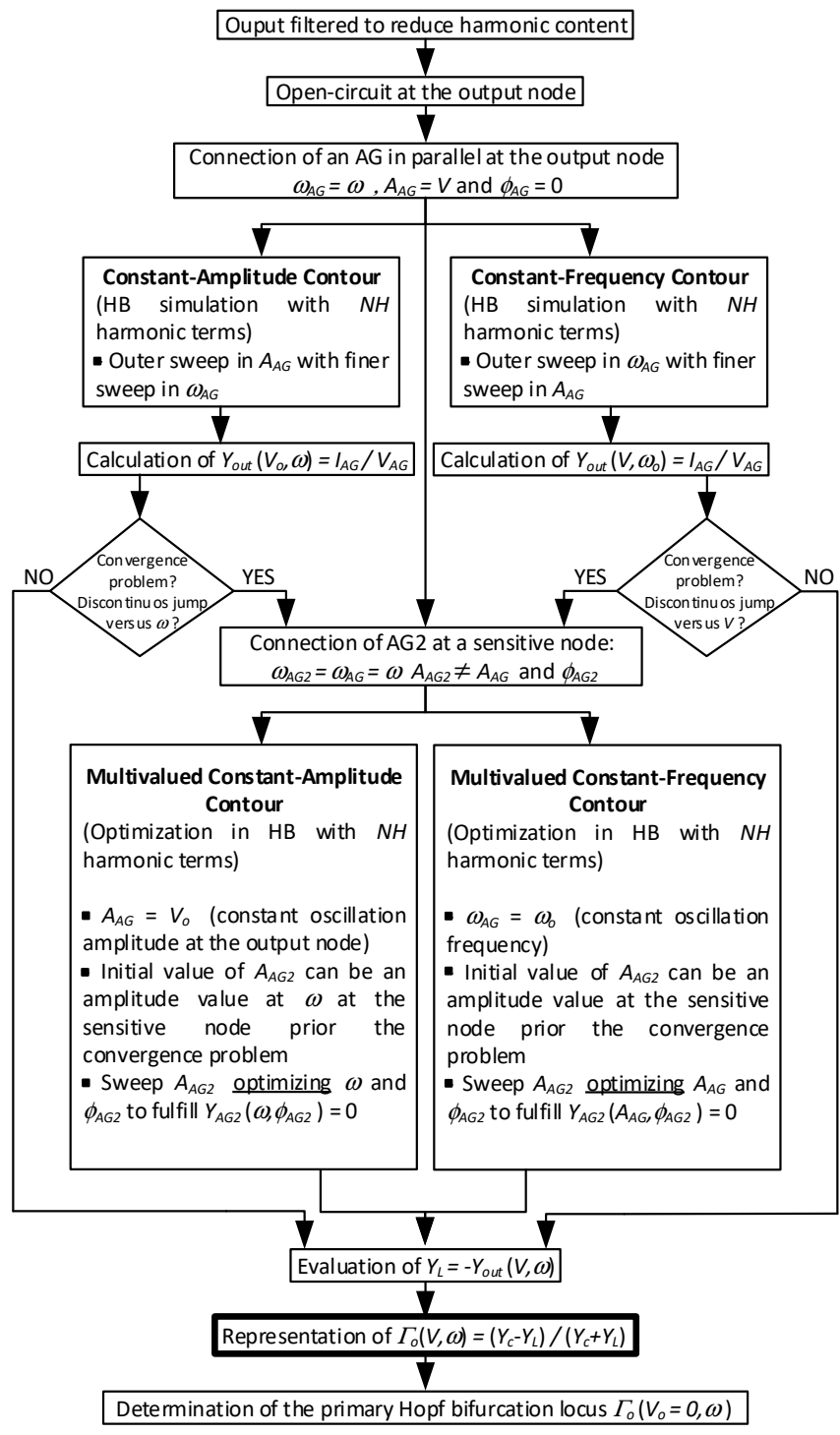

Fig. 5. Flowchart showing the different stages in the analysis of the impact of the termination load on the oscillator solution. The procedures for the calculation of the constant-amplitude and constant-frequency frequencies, including the primary Hopf locus, are indicated.

Fig. 6 and Fig. 7(a) present the constant-amplitude and constant-frequency contours, respectively, of the oscillator circuit in Fig. 4, obtained in $\mathrm{HB}$ with $\mathrm{NH}=8$. In single-valued situations [as is the case for most of the reflection coefficients $\Gamma_{o}$ in Fig. 6] each curve $\Gamma_{o}\left(V_{o}, \omega\right)$ in Fig. 6 constitutes a boundary between two regions of the Smith chart, one region providing an oscillation amplitude higher than $V_{o}$ and the other providing an oscillation amplitude smaller than $V_{o}$. Though most of the constant amplitude or frequency contours can be directly calculated through a simple sweep, without any optimization procedures, convergence failure will be encountered in the case of multivalued curves. This problem is described in detailed in subsection III.B.

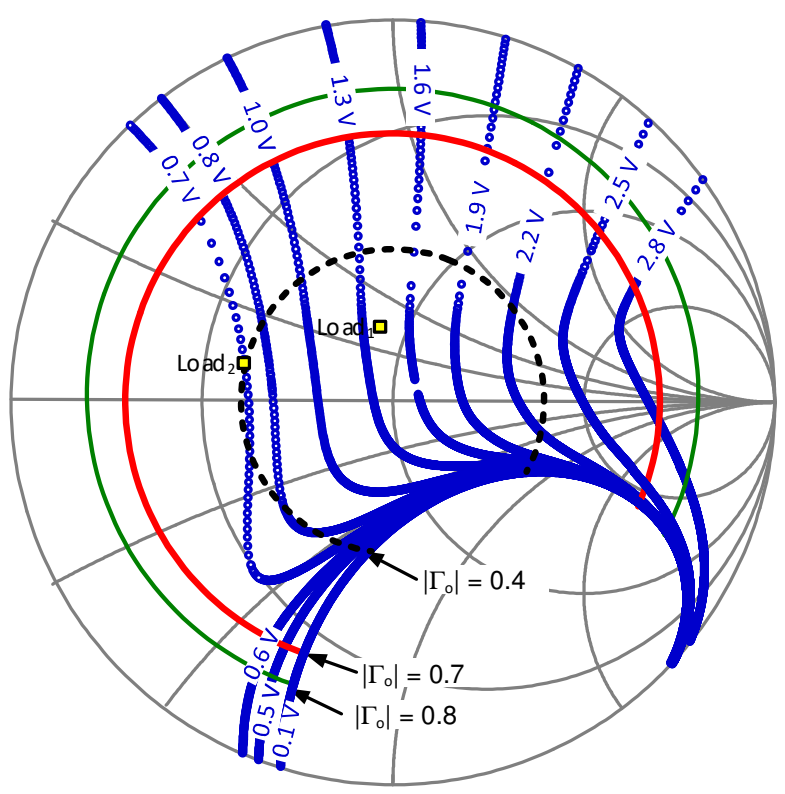

Fig. 6. Constant amplitude contours. The three solution paths considered for an independent validation are indicated. They correspond to the three constant reflection-coefficient magnitudes $\left|\Gamma_{o}\right|=0.4, \quad\left|\Gamma_{o}\right|=0.7$, and $\left|\Gamma_{o}\right|=0.8$.

Constant output-power contours have also been obtained by processing the data in the constant-frequency contours, in which a finer amplitude sweep in $A_{A G}$ is carried out. They are represented in Fig. 7(a). The exemplary curves in the inset show the variation of output power through two constant oscillation-frequency contours, traced versus the real part of the load impedance $\operatorname{Re}\left(Z_{L}\right)$. Note that the imaginary part $\operatorname{Im}\left(Z_{L}\right)$ also varies through each curve and is different at each point. As can be seen, the output power decreases fast when approaching the Hopf bifurcation (oscillation extinction) and the boundary of the Smith chart (zero-resistance load). In fact, near this boundary and near the Hopf locus, each constantfrequency contour passes through a wide interval of outputpower values. Thus, the constant output-power contours are denser in these two regions, both in simulation and measurement. The circuit has been experimentally characterized using a triple-stub tuner, which replaces the 50 $\Omega$ load. Constant-output power measurements, obtained when varying the lengths of the tuner, are superimposed in Fig. 7(a). The experimental constant-frequency contours are shown in Fig. 7(b). As can be seen, the oscillation bandwidth is narrower in comparison with the simulation predictions, which is attributed to inaccuracies in the modeling of the oscillator elements. Nevertheless, there is a very good qualitative agreement. Fig. 7(c), (d) and (e) present several experimental spectra in the constant-frequency contours corresponding to the oscillation frequencies $f_{o}=2.4 \mathrm{GHz}, f_{o}=2.437 \mathrm{GHz}$ and $f_{o}=2.47 \mathrm{GHz}$. In the three cases, the measured fundamental frequency output power remains nearly constant, in consistency with the contour predictions. 


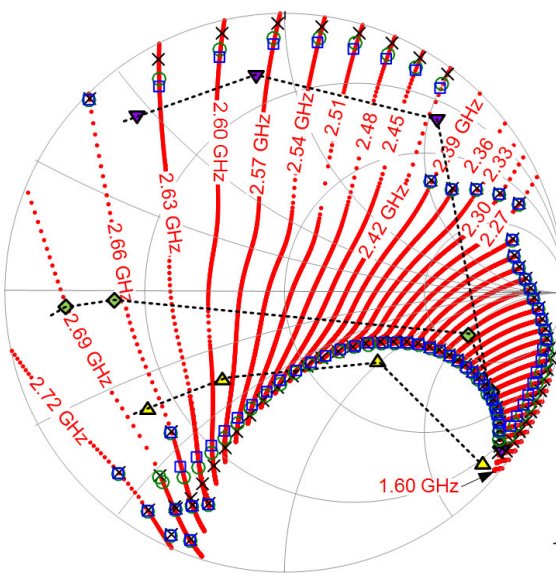

(a)

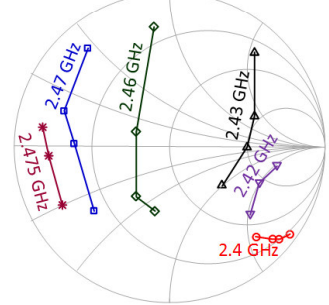

(b)

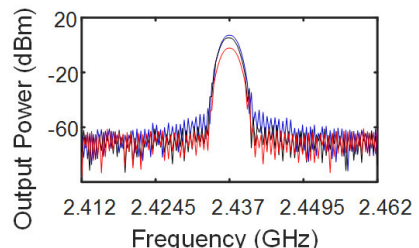

(d)
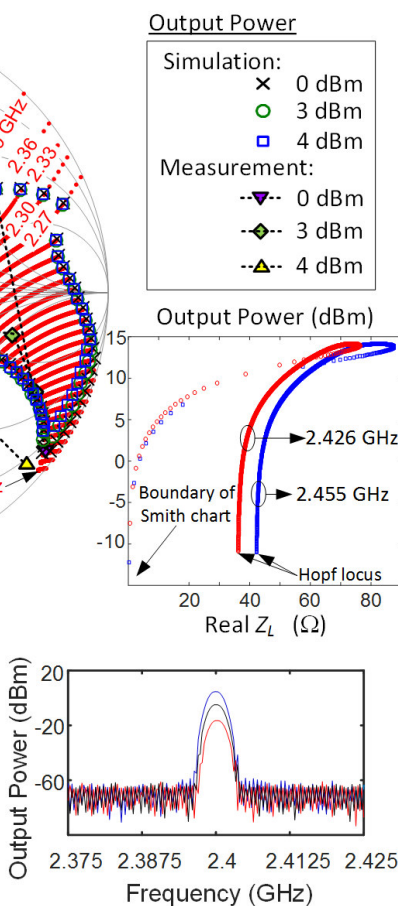

(c)

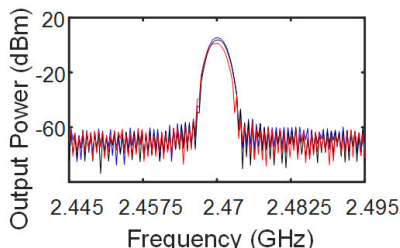

(e)
Fig. 7. Constant oscillation-frequency contours. (a) Simulated with the method in III.A. Constant output-power contours for $0 \mathrm{dBm}, 3 \mathrm{dBm}$ and 4 $\mathrm{dBm}$ are also represented, with measurements superimposed. (b) Experimental constant-frequency contours. Inset: variation of output power through two constant-frequency contours, traced versus the real part of the load impedance $\operatorname{Re}\left(Z_{L}\right)$. The imaginary part $\operatorname{Im}\left(Z_{L}\right)$ is different at each curve point. (c) Experimental spectra in the constant-frequency contour corresponding to the oscillation frequency $f_{o}=2.4 \mathrm{GHz}$. (d) Spectra corresponding to $f_{o}=2.437 \mathrm{GHz}$. (e) Spectra corresponding to $f_{o}=2.47 \mathrm{GHz}$.

\section{A. Oscillation Extinction}

As gathered from Fig. 6, there is a region of the output Smith chart which is void of oscillation curves. This region is delimited by a degenerate constant-amplitude curve, at which the oscillation amplitude tends to zero: $\Gamma_{o}\left(V_{o}=0, \omega\right)$. This curve constitutes an oscillation boundary, agreeing with the primary Hopf bifurcation locus [24], [27]-[28], [37]-[38], traced in the Smith chart corresponding to the termination load $\Gamma_{o}$ (see flowchart of Fig.5). At the primary Hopf bifurcation locus, the oscillation is either generated or extinguished, depending on the sense of variation of the two parameters, which in this case agree with the amplitude $\rho_{o}$ and phase $\phi_{o}$ of the reflection coefficient $\Gamma_{o}$. If undesired mismatch effect leads the termination load to this empty region of Fig. 6 (without any constant-amplitude curves), the oscillation will be extinguished. Note that the steady-state oscillation amplitude progressively decreases when approaching the Hopf bifurcation locus. This gradual extinction may take place at either one or the other side of the primary Hopf locus, depending on the supercritical or subcritical nature of this bifurcation [37]-[38].

\section{B. Multivalued Constant-Amplitude or Constant-Frequency} Contours

Nonlinear circuits are susceptible to exhibit multi-valued solution curves when varying a circuit parameter [37]-[38]. In the analysis performed here, these multi-valued solutions may be encountered when connecting the auxiliary generator (AG1) at the output port to obtain the function $Y_{\text {out }}(V, \omega)$. Note that AG1, with amplitude $A_{A G 1}=V$ and frequency $\omega_{A G 1}=\omega$, constitutes an independent excitation, so in this simulation $V$ and $\omega$ are not acting as state variables, but as parameters of the excitation source. Two or more steady-state solutions, with different values of the circuit internal voltages and currents, may coexist for a same pair of excitation quantities $\left(V_{o}, \omega_{b}\right)$. As a result, different values of the output-admittance function $Y_{\text {out }}\left(V_{o}, \omega_{o}\right)$ may be obtained for a same excitation pair $V_{o}, \omega_{o}$.

The multi-valued region (in terms of any internal variable) is delimited by infinite-slope points, or turning points, at which the HB Jacobian matrix becomes singular [24], [27][28]. Thus, the problem is easily detected in commercial HB since it will either lead to a convergence problem or give rise to a discontinuous jump versus $\omega$, in a constant-amplitude contour, calculated from $Y_{\text {out }}\left(V_{o}, \omega\right)$, or a discontinuous jump versus $V$, in a constant-frequency contour, calculated from $Y_{\text {out }}\left(V, \omega_{o}\right)$. In the circuit in Fig. 4, multivalued responses $Y_{\text {out }}\left(V_{o}, \omega\right)$ are obtained for relatively large $V_{o}$.

The described multi-valued curves can be easily traced, with the aid of a second auxiliary generator, denoted as AG2 [Fig. 4(c)], which enables the implementation of a parameterswitching procedure in commercial HB [28]. See flowchart of Fig.5. The auxiliary generator AG2 should be connected to a sensitive analysis node, such as a device terminal [28]. It is a standard auxiliary generator, which should fulfill a nonperturbation condition of the steady-state oscillatory solution [28], [35]. The amplitude of this generator will be $A_{A G 2}$, and different from that of the auxiliary generator (AG1) connected to the termination node. Its frequency will be the same, and given by $\omega_{A G 2}=\omega_{A G}=\omega$. Because the two auxiliary generators operate at the same frequency and the phase of the output auxiliary generator (AG1) had been arbitrarily set to zero, the phase of AG2, $\phi_{A G 2}$, will be an unknown of the problem.

The auxiliary generator AG2 is used to obtain the multivalued sections of $Y_{\text {out }}\left(V_{o}, \omega\right)$ or $Y_{\text {out }}\left(V, \omega_{o}\right)$ through optimization in commercial HB. Let the case of a multivalued constant-amplitude contour, derived from $Y_{\text {out }}\left(V_{o}, \omega\right)$, be considered. The amplitude of AG1 (the auxiliary generator that fixes the oscillation amplitude at the output termination) is set to $A_{A G}=V_{o}$ and kept constant at this value. An initial guess for the amplitude and frequency of AG2 can be obtained from those corresponding to any successful point in the original calculation through (4), prior to the convergence problem. The 
initial value of $A_{A G 2}$ is obtained by measuring the voltage amplitude at $\omega$, at the internal node where AG2 will be connected. Then, starting from this initial value, $A_{A G 2}$ will be swept up or down, optimizing $\omega$ and $\phi_{A G 2}$ at each sweep step, in order to fulfill the non-perturbation condition:

$$
Y_{A G 2}\left(\omega, \phi_{A G 2}\right)=\frac{I_{A G 2}}{A_{A G 2}}=0
$$

Fig. 8 illustrates the application of the above method to obtain the multivalued constant-amplitude contour corresponding to $V_{o}=0.7 \mathrm{~V}$. It presents the variation of the oscillation amplitude at the drain node [Fig. 8(a)] versus the excitation frequency $\omega_{A G}=\omega_{A G 2}=\omega$ for $V_{o}=0.7 \mathrm{~V}$. The curve exhibits a multi-valued $\omega$ interval with three coexisting solutions, each providing a distinct $Y_{\text {out }}\left(V_{o}, \omega\right)$, shown in Fig. 8(b). This multi-valued $\omega$ interval is delimited by two turning points, at $\mathrm{TP}_{1}=2.626 \mathrm{GHz}$ and $\mathrm{TP}_{2}=2.646 \mathrm{GHz}$.

With the aid of the second auxiliary generator AG2, it is possible to complete the constant output-amplitude contours $\Gamma_{o}\left(V_{o}, \omega\right)$ of a given oscillator design, as has been done in Fig. 6. An analogous procedure should be applied to complete the constant-frequency contours $\Gamma_{o}\left(V, \omega_{o}\right)$, as done in Fig. 7(a).

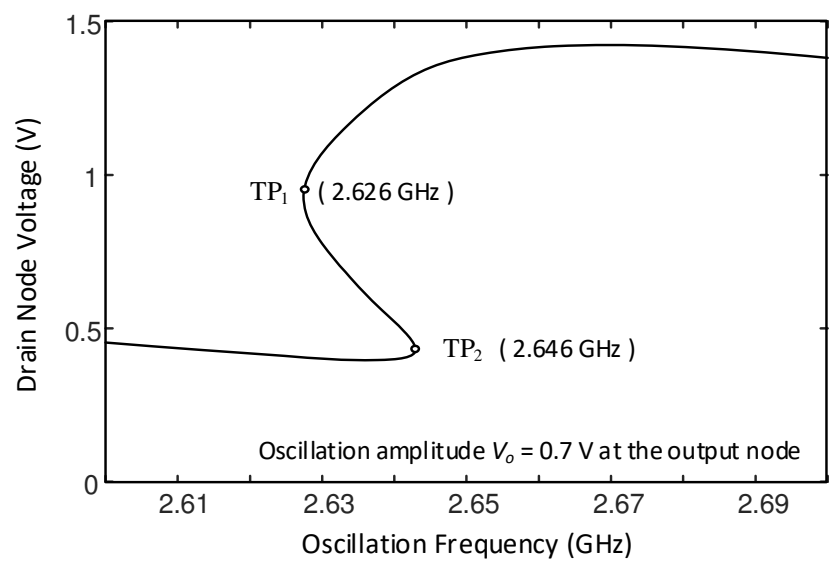

(a)

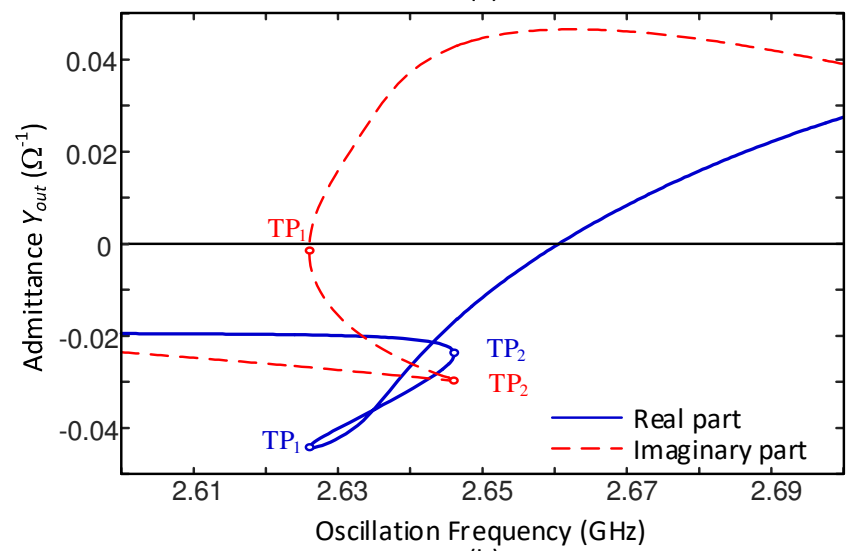

(b)

Fig. 8. Multi-valued constant-amplitude contour, corresponding to the firstharmonic amplitude $V_{o}=0.7 \mathrm{~V}$. (a) Variation of the oscillation amplitude at the drain node under a constant excitation amplitude $A_{A G 1}=V_{o}=0.7 \mathrm{~V}$ at the circuit output node, represented versus the excitation frequency in the interval $2.6 \mathrm{GHz}$ to $2.7 \mathrm{GHz}$. (b) Variation of the admittance $Y_{\text {out }}$, calculated through (4) for the same excitation amplitude and frequency interval.

\section{Independent Validations}

Validations of the new method with independent HB simulations should be the most reliable, since this way there cannot be any possible discrepancies due to inaccurate component models. The first validation is based on the implementation of particular loads of the Smith chart, followed by an oscillator analysis with the default method existing in the commercial HB software. The loads considered are indicated with squares in Fig. 6. The first load $\operatorname{Load}_{1}$ has been implemented with a resistor connected in series with an inductor, of values $R=43.5 \Omega$ and $L=1.1 \mathrm{nH}$. The analysis is carried out under the same number of harmonic components, $N H=8$, considered when obtaining the constant-amplitude contours of Fig. 6. According to these contours the oscillation amplitude should have a value comprised between $1.3 \mathrm{~V}$ and $1.6 \mathrm{~V}$. This is total agreement with the output amplitude spectrum shown in Fig. 9. Note that due to the secondharmonic trap, the second harmonic amplitude is very low. Other harmonic components exhibit low amplitudes too, which is consistent with the excellent agreement between the predictions of Fig. 6 and the results of Fig. 9. In a second test, Load $_{2}$ has been implemented using also an inductor in series with a resistor of values $R=22 \Omega$ and $L=0.39 \mathrm{nH}$. Because this load is located on the output-amplitude contour $V_{o}=0.7$ $\mathrm{V}$, the independent simulation of the spectrum should exhibit this first harmonic amplitude, which has also been verified, as shown in Fig. 9.

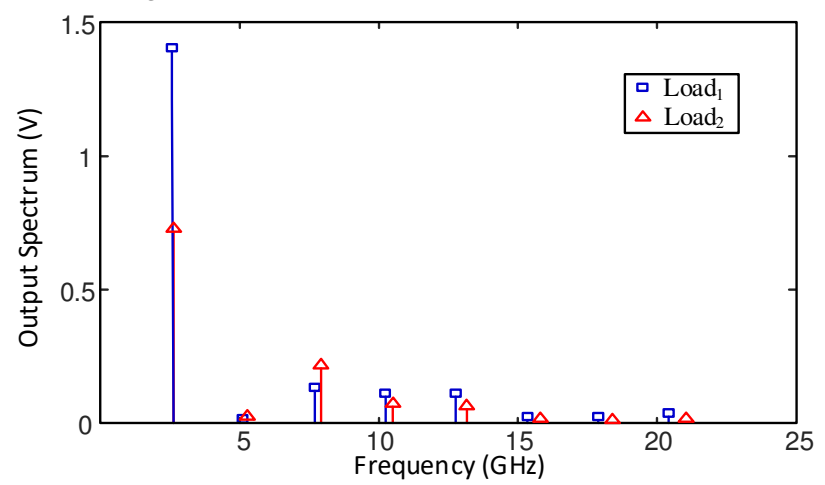

Fig. 9. Validation of oscillation amplitudes for the two loads indicated with squares in Fig. 6 and denoted as $\operatorname{Load}_{1}$ and $\operatorname{Load}_{2}$, respectively. $\operatorname{Load}_{1}$ is implemented with the series connection of a resistor $R=43.5 \Omega$ and inductor $L=1.1 \mathrm{nH}$, and $\mathrm{Load}_{2}$ is implemented with the series connection of $R=22 \Omega$ and $L=0.39 \mathrm{nH}$.

The second validation is a global one, and based on the calculation of a whole oscillation-solution curve, obtained by following a certain path in the $\Gamma_{\mathrm{o}}$ Smith chart. Note that physically following this particular path would involve synthesizing, at each frequency $\omega$, the reflection coefficient $\Gamma_{o}(\omega)$ corresponding to that point of the path. The three paths considered are circles in the Smith chart, defined as $\left|\Gamma_{o}\right|=0.4$, $\left|\Gamma_{o}\right|=0.7$ and $\left|\Gamma_{o}\right|=0.8$ (Fig. 6). In Fig. 10(a), the resulting variations of the oscillation amplitude have been represented versus the phase $\phi_{o}$ of the output reflection coefficient. In all cases, the oscillation amplitude grows from zero and is extinguished to zero. In consistency with this, the constant magnitude circle $\left|\Gamma_{o}\right|$ crosses more than once the same 
constant-amplitude curve in Fig. 6. In the three cases, the oscillation amplitude reaches a zero value at the phase $\phi_{o}$ for which the corresponding circle hits the primary Hopf locus $\Gamma_{o}\left(V_{o}=0, \omega\right)$. The case of the circle $\left|\Gamma_{o}\right|=0.8$, providing a multi-valued amplitude curve versus $\phi_{o}$, is discussed in the next section.
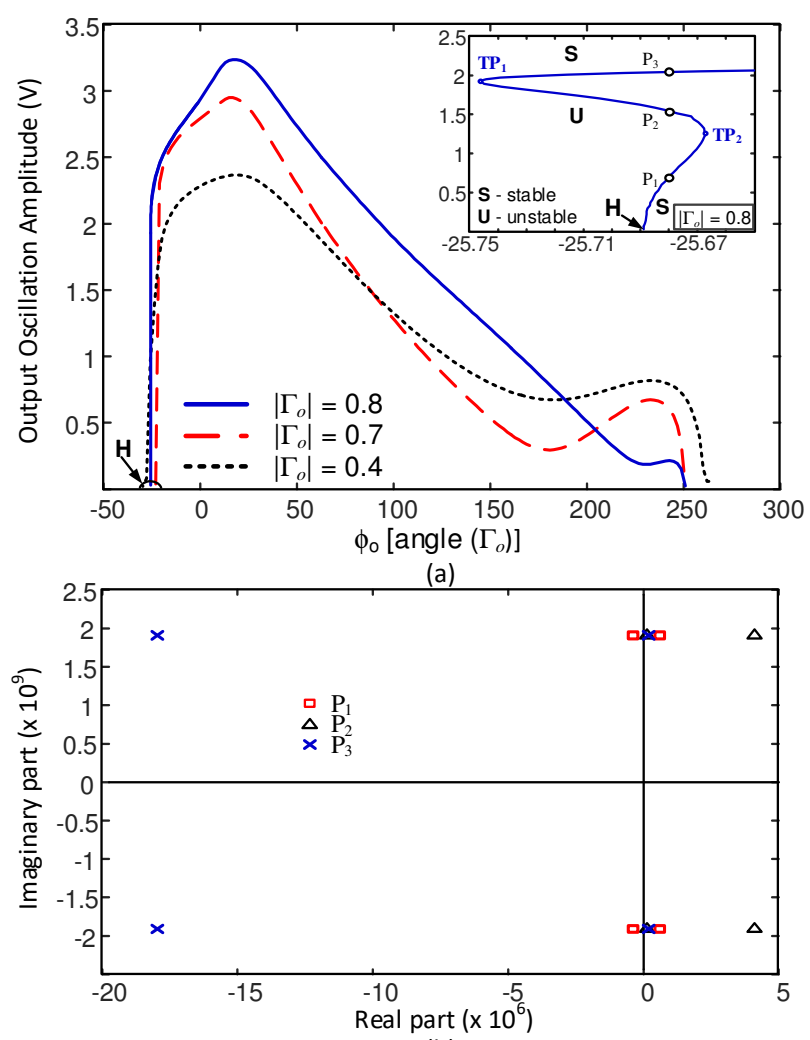

(b)

Fig. 10. Variation of the output oscillation amplitude when following the reflection-coefficient circles defined by the magnitudes $\left|\Gamma_{o}\right|=0.4,\left|\Gamma_{o}\right|=0.7$ and $\left|\Gamma_{o}\right|=0.8$, respectively, traced versus the reflection-coefficient phase $\phi_{o}$. (a) Solution curve. (b) Stability analysis of the three coexisting oscillation points $\mathrm{P}_{1}, \mathrm{P}_{2}$ and $\mathrm{P}_{3}$ under short and open circuit terminations for $\left|\Gamma_{o}\right|=0.8$.

A third independent validation relies on the use of a physical load. For illustration, two different passive loads have been considered (Fig. 11). One consists of the parallel connection of a resistor $R=100 \Omega$ and an inductor $L$. Under variations of $L$, this load gives rise to the reflection-coefficient path "A", shown in the inset of Fig 11(a). In that figure, the resulting variation of the oscillation amplitude $V$ has been represented versus the oscillation frequency $\omega$, and the reflection coefficients $\Gamma_{\mathrm{o}}$ exhibited by the load at various points of the curve are indicated. Note that this solution curve has been calculated through an independent oscillator analysis, using $N H=8$ harmonic terms. The values of the oscillation amplitude and frequency are in full agreement with the predictions of Fig. 6 and Fig. 7(a). The second load consists of the series connection of a resistor $R=50 \Omega$ and a capacitor $C$, which, under variations of the capacitor value, gives rise to the reflection-coefficient path "B" represented in the inset of Fig. 11(b). The resulting variation of the oscillation amplitude $V$ has been represented versus the oscillation frequency $\omega$, and the reflection coefficients exhibited by the load at various points of the curve are indicated. As in the previous case, the values are in full agreement with the predictions of Fig. 6 and Fig. 7(a). Fig. 11(c) presents the simulated oscillation frequency and output power when varying the capacitor as in Fig. 11(b). The predicted frequency and power are compared with measurements results, obtained by changing the capacitor from $10 \mathrm{pF}$ to $2 \mathrm{pF}$. As can be seen there is a very good agreement.
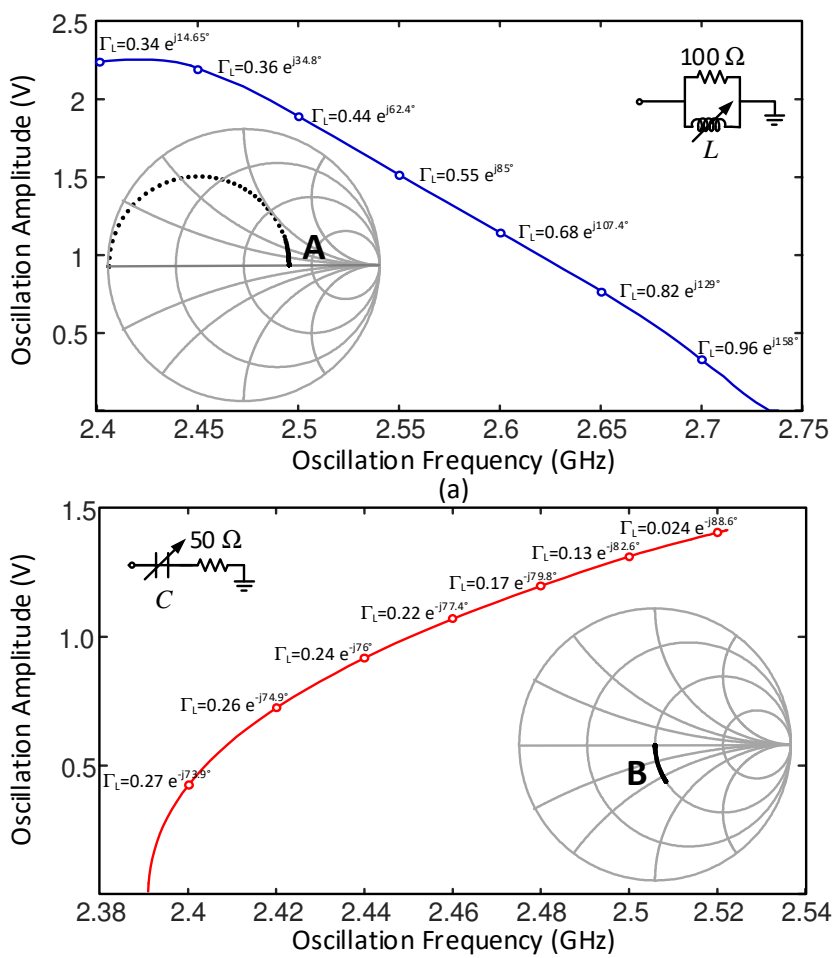

(b)

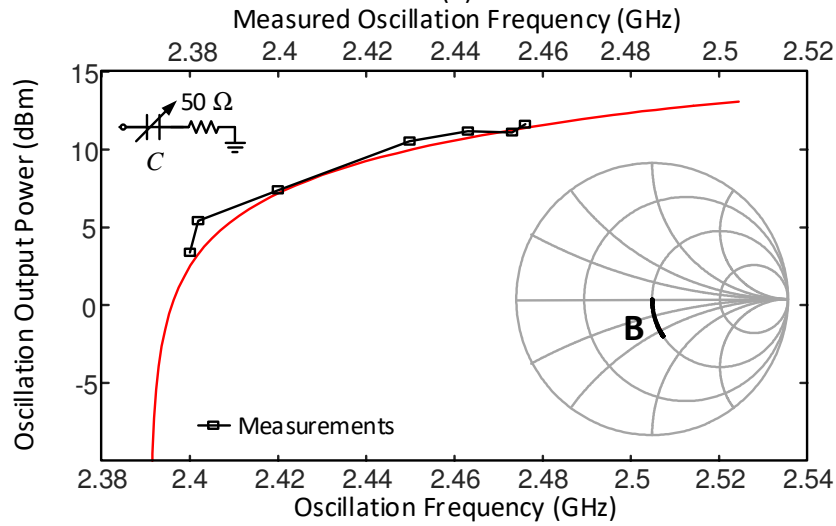

(c)

Fig. 11. Independent validation using physical loads. (a) Parallel connection of a resistor $R=100 \Omega$ and an inductor $L=13.5 \mathrm{nH}$. Representation of the oscillation amplitude versus the oscillation frequency when varying the inductor $L$. The reflection coefficient associated with this load follows the path "A" represented in the inset of the figure. (b) Series connection of a resistor $R=50 \Omega$ and a capacitor $C=10 \mathrm{pF}$. Representation of the oscillation amplitude versus the oscillation frequency when varying the capacitor $C$. The reflection coefficient associated with this load follows the path " $\mathrm{B}$ " in the inset of the figure. (c) Experimental validation. Oscillation output power and frequency when varying the capacitor as in Fig. 11(b). Simulations are compared with measurements results. 


\section{Stability ANALYSIS}

The analysis in Fig. 6 and Fig. 7 predicts the effect of the termination impedance on the steady-state oscillation frequency and output power. However, the resulting oscillatory solutions may be stable (physical) or unstable. Different instability phenomena may be observed under the variation of the output load. These include the hysteresis [24], [27]-[28], [37]-[38], and sudden oscillation extinctions, as well as the onset of incommensurable or subharmonic oscillations.

\section{A. Hysteresis}

As discussed in Section III.B, multi-valued solution curves often arise in nonlinear circuits under a strong excitation, as was the case of the contours $\Gamma_{o}\left(\mathrm{~V}_{o}, \omega\right)$ or $\Gamma_{o}\left(\mathrm{~V}, \omega_{o}\right)$, when calculated with a high amplitude of AG1. The multi-valued solutions are particularly common in oscillator circuits, since the active-device negative conductance often exhibits a nonmonotonous variation with the excitation amplitude [39]. In fact, the device conductance may become more negative in some amplitude intervals, though finally increasing, from a certain amplitude value [39], as expected in physical devices. The multivalued behavior is often observed near the oscillation edges (primary Hopf bifurcations), as shown in Fig. 10(a).

Multi-valued responses versus the reflection coefficient $\Gamma_{o}$ can be easily detected through a simple inspection of the geometry of the constant-amplitude contours in the Smith chart. In the particular case of the circuit in Fig. 4, this occurs in the region near the primary Hopf bifurcation locus, where the oscillation amplitude $V$ tends to zero $(V \rightarrow 0)$. This is in agreement with the solution curve obtained when following the circle $\left|\Gamma_{o}\right|=0.8$, shown in the inset of Fig. 10(a). In a small interval of reflection-coefficient phase $\phi_{o}$ comprised between $25.74^{\circ}$ and $-25.67^{\circ}$, the steady-state oscillation curve is multivalued. This multi-valued interval is delimited by two turning points $\mathrm{TP}_{1}$ and $\mathrm{TP}_{2}$. However, one should take into account that coexisting solutions may also arise as a result of the particular frequency characteristic of the termination load $\Gamma_{L}(\omega)$, where $\omega=2 \pi f$. If this load takes the values $\Gamma_{L}\left(\omega_{1}\right)=\Gamma_{o}\left(V_{1}, \omega_{1}\right)$ and $\Gamma_{L}\left(\omega_{2}\right)=\Gamma_{o}\left(V_{2}, \omega_{2}\right)$, two steady-state oscillatory solutions at the respective amplitudes and frequencies $V_{1}, \omega_{1}$ and $V_{2}, \omega_{2}$ [16], will coexist for the same circuit-element values. Detection of this solution coexistence requires a knowledge of the frequency-dependent function $\Gamma_{L}(\omega)$.

\section{B. Stability Analysis}

The prediction of the stability properties of a given oscillatory solution involves the consideration of a small perturbation at a frequency $f$, incommensurable with the oscillation frequency $\omega_{b}=2 \pi f_{o} \quad$ [12]-[14]. This small perturbation is also considered in the potential-stability analysis of PAs, described in Section II. However, a direct extension of the PA analysis to oscillator circuits is not possible. The PA potential-stability analysis aims at predicting the possible observation of negative resistance at any of the three relevant sideband frequencies $f_{b}=f, f_{l}^{*}=-f_{\text {in }}+f$ and $f_{u}=f_{\text {in }}+f$, under arbitrary passive terminations at the other two sidebands. Such a negative resistance is undesired in PA and would give rise to oscillations under certain passive-loading conditions. In the oscillator case, the negative resistance [exhibited by the active device(s)] is required to self-sustain the periodic solution at $f_{o}$. However, the active device(s) will not only exhibit this negative resistance at the particular oscillation frequency $f_{o}$, but in a certain frequency band about $f_{o}$. The potential instability analysis in [11] and Section II assumes a discrete termination at $\Gamma_{o}$ and arbitrary terminations of the sideband frequencies. Thus, it will obviously detect the negative resistance about $f_{o}$ that is inherent to the oscillator behavior.

Without any knowledge of the frequency characteristic of the oscillator load $\Gamma_{L}(f)$, the only detectable potentialinstabilities will be those associated to the change in the nonlinear-circuit operation conditions, as a result of a variation in the termination load $\Gamma_{o}$ at the fundamental frequency $f_{o}$. For each $\Gamma_{o}$ fulfilling the steady-state oscillation condition (5)-(6), this potential instability can be predicted through pole-zero identification [12]-[14], applied in the two limit cases of open and short circuit terminations at the three relevant sidebands $f_{b}=f, f_{l}^{*}=-f_{o}+f$ and $f_{u}=f_{o}+f$. The availability of the constantamplitude and constant-frequency contours (such as the ones presented in Fig. 6 and Fig. 7(a)) will greatly facilitate this analysis procedure.

The stability analysis is based on the connection of an additional small-signal current source $I(f)$ at the incommensurable frequency $f[12]-[14]$, which is used to calculate a closed-loop transfer function, given by the ratio between the source voltage and current $Z(f)=V(f) / I(f)$. For each pair of values $A_{A G 2}=V_{o}$ and $\omega_{A G 2}=\omega$, the frequency $f$ is swept and a large-signal small-signal analysis (or conversionmatrix approach) is performed. The stability properties are determined through the application of pole-zero identification to $Z(f)$. This kind of analysis should be able to detect unstable complex-conjugate poles at the oscillation frequency $f_{o}$, observed when passing through a turning point [28]. It should also be able to detect a pair of complex-conjugate poles at an incommensurable frequency $f_{o}$, (giving rise to a doublyautonomous regime) or at the divided-by-two frequency $f_{o} / 2$ (giving rise to a subharmonic regime).

For illustration, pole-zero identification has been applied through the multi-valued oscillation curve in the inset of Fig. 10(a), under the limit conditions of open and short circuit at the three relevant sideband frequencies. This should enable a detection of "internal instabilities", independent of the frequency characteristic $\Gamma_{L}(f)$ of the output load. Fig. 10(b) shows the pole loci for the three coexisting solution points $\mathrm{P}_{1}$, $\mathrm{P}_{2}$ and $\mathrm{P}_{3}$, for open-circuit and short circuit terminations at the sidebands. Results are overlapped in the three cases. For the three solution points $\left(\mathrm{P}_{1}, \mathrm{P}_{2}\right.$ and $\left.\mathrm{P}_{3}\right)$, there is a pair of complex conjugate poles at the oscillation frequency on the imaginary axis, which is due to the autonomous nature of periodic solution [21]-[22]. Due to numerical limitations, this pair of poles may be slightly shifted from the imaginary axis. In addition to this pair of poles, the points $\mathrm{P}_{1}$ and $\mathrm{P}_{3}$ exhibit a pair of poles at $f_{o}$ located on the left-hand side (LHS) of the complex plane, so these two solutions are stable. The point $\mathrm{P}_{2}$ exhibits an additional pair of complex-conjugate poles at $f_{o}$ 
located on the right-hand side (RHS) of the complex plane, so this solution is unstable. The oscillation-curve section between $\mathrm{H}$ and $\mathrm{TP}_{1}$ is stable. The section between $\mathrm{TP}_{1}$ and $\mathrm{TP}_{2}$ is unstable with one real pole on the right hand side of the complex plane. The upper section is stable. However, the frequency characteristic of the output load $\Gamma_{L}(f)$ will generally affect the stability properties. This is illustrated in Fig. 12, where the output reflection coefficient $\Gamma_{o}=0.4 e^{j 120 \circ}$ at the fundamental frequency $f_{o}=2.598 \mathrm{GHz}$ has been implemented in three different manners.

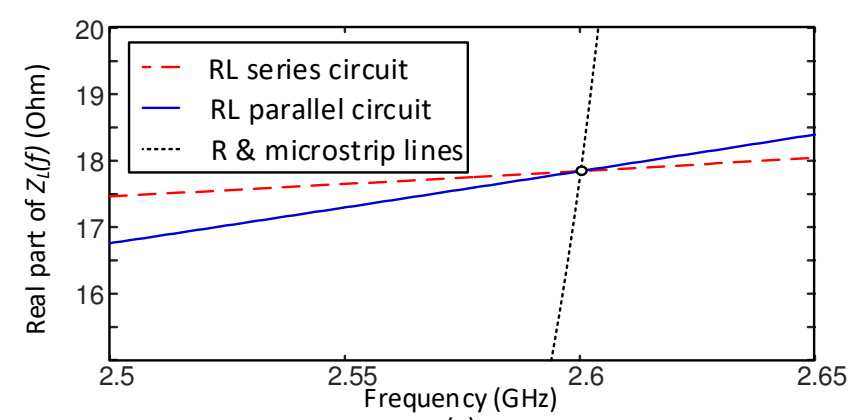

(a)

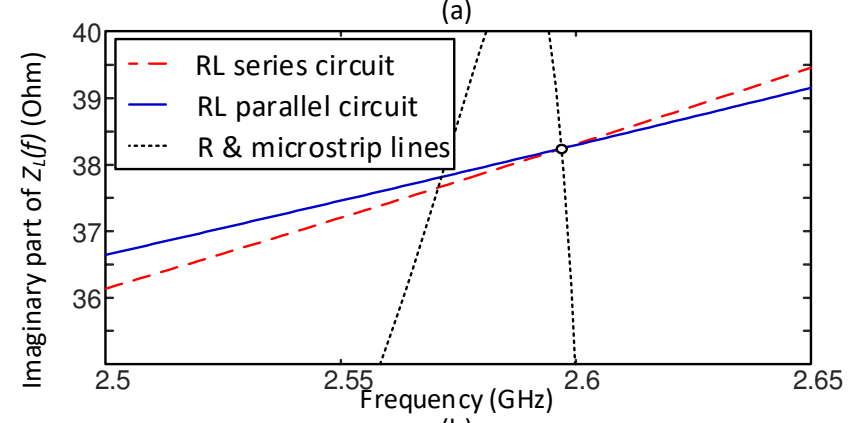

(b)

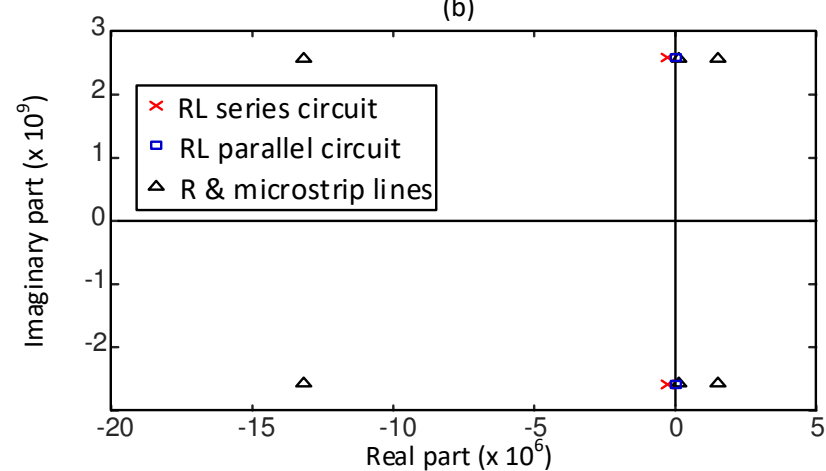

(c)

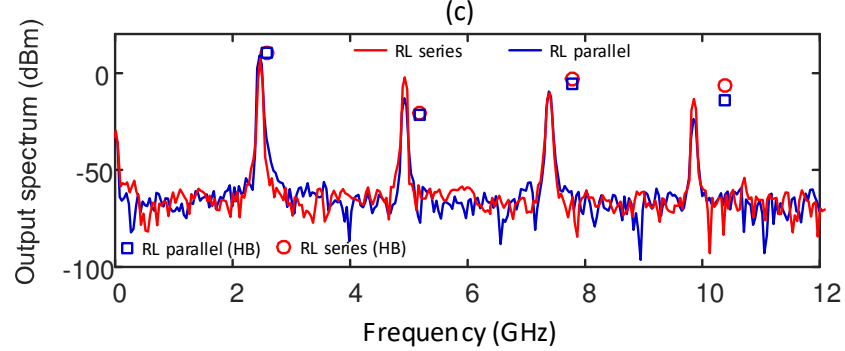

(d)

Fig. 12. Stability properties for three different implementations of $\Gamma_{o}=0.4 e^{j 120^{\circ}}$ at $f_{o}=2.598 \mathrm{GHz}$. (a) Expanded view of the real part of $Z_{L}(f)$ about the free-running frequency $f_{o}$, in the three cases. (b) Expanded view of the imaginary part. (c) Pole loci obtained through pole-zero identification of the transfer function $Z(f)$ in the three cases. (d) Comparison of the simulated and measured spectra obtained with for the two stable implementations.
The three different implementations are based on the series and parallel connections of a resistor and inductor and on the use of microstrip lines. Fig. 12(a) and Fig. 12(b) show an expanded view of the real and imaginary parts of the load impedance $Z_{L}(f)$ about the free-running frequency $f_{o}$, in the three cases. As can be seen, the impedance value at $f_{o}$ is identical for the three implementations. However, the frequency characteristic is different. Identification of the transfer function $Z(f)$ provides stable behavior in the series and parallel R-L circuits and unstable behavior in the microstrip implementation, as shown in Fig. 12(c). Fig 12 (d) compares the simulated and measured spectra obtained with for the two stable implementations.

\section{PHASE NOISE}

Phase noise is an undesired but unavoidable characteristic of oscillator circuits, which degrades the oscillator signal and may cause demodulation errors [29]-[30]. Due to the dependence of the oscillatory solution on the termination load, one can expect this load to have an impact on the oscillator phase-noise spectral density. To illustrate this, the phase noise of the two designs obtained with the two stable load circuits of Section IV.B (Fig. 12) will be analyzed.

One load is implemented with a R-L series circuit presenting an impedance $Z_{s}(f)$ and the other one is implemented with a R-L parallel circuit, with impedance $Z_{p}(f)$. The $R$ and $L$ components of both loads have been set to fulfill $Z_{s}\left(f_{o}\right)=Z_{p}\left(f_{o}\right)$, where $f_{o}$ is the oscillation frequency, therefore the two loads provide the same oscillation frequency and output power at the fundamental frequency. Note that this equality is not fulfilled in general for $f \neq f_{o}$.

In the first place, for the phase-noise analysis, the conversion matrix approach [32]-[33] has been used, including all the noise sources existing in the oscillator circuit. The resulting spectra are shown in Fig. 13. As can be seen, the phase noise characteristics obtained for both loads disagree, since they are influenced by different frequency-dependent loads. The conversion matrix approach does not take into account the inherent nonlinearity of the oscillator circuit with respect to phase deviations. As a consequence, the near carrier predicted spectrum will be inaccurate. In order to refine the near carrier spectrum, at a second stage, the phase-noise analysis method presented in [8],[31] has been applied. In this method, the phase noise process is assumed to be unbounded, and the power spectral density (PSD) of the output voltage about the first harmonic component is calculated as:

$$
\begin{aligned}
& S(f) \cong F\{R(\tau)\}, R(\tau)=\exp \left\{-\frac{\sigma^{2}(|\tau|)}{2}\right\}, \\
& \sigma^{2}(t, \tau)=\left\langle[\Delta \phi(t+\tau)-\Delta \phi(t)]^{2}\right\rangle \cong \sigma^{2}(|\tau|)
\end{aligned}
$$

where $\Delta \phi(t)$ is the first harmonic phase perturbation, $F$ is the Fourier transform operator and $f$ is the frequency offset from the carrier. It can be demonstrated [31] that, even in the presence of flicker noise sources, in most practical measurement procedures the variance $\sigma^{2}(t, \tau) \sigma^{2}(t, \tau)$ can be associated to a nearly-stationary process and then the time 
dependence on $t$ can be neglected. The phase perturbation $\Delta \phi(t)$ is a stochastic process that can be determined by the following procedure. First, the effect of all the noise sources presented in the circuit is equated by a single current source $i_{n}(t)=\operatorname{Re}\left\{I_{n}(t) e^{j 2 \pi f_{o} t}\right\}$ placed at an observation node. The time varying nature of the harmonic component is due to the effect of the noise sources. Then, following the perturbation analysis in [31], the first harmonic phase perturbation is given by the following equation in the Laplace domain:

$$
\Delta \phi(s)=\frac{1}{s} c(s) I_{n}(s)
$$

where $c(s)$ is a transfer function containing the poles resulting from the stability analysis of the oscillating solution. Assuming that the time-varying harmonic $I_{n}(t)$ is modulated by both white and colored noise sources, the variance $\sigma^{2}(|\tau|)$ can be determined. Finally, this variance function is used to obtain the phase noise spectrum from (5). This has been done in Fig. 13, obtaining two phase noise characteristics that agree with the conversion matrix predictions for frequency offset values far enough from the carrier. The near carrier spectrum flattening assures that the total power about the first harmonic component is bounded. Experimental measurements of the phase noise spectrum have been superimposed for the two different loads $Z_{s}(f)$ and $Z_{p}(f)$. Very good agreement is obtained with the simulation results.

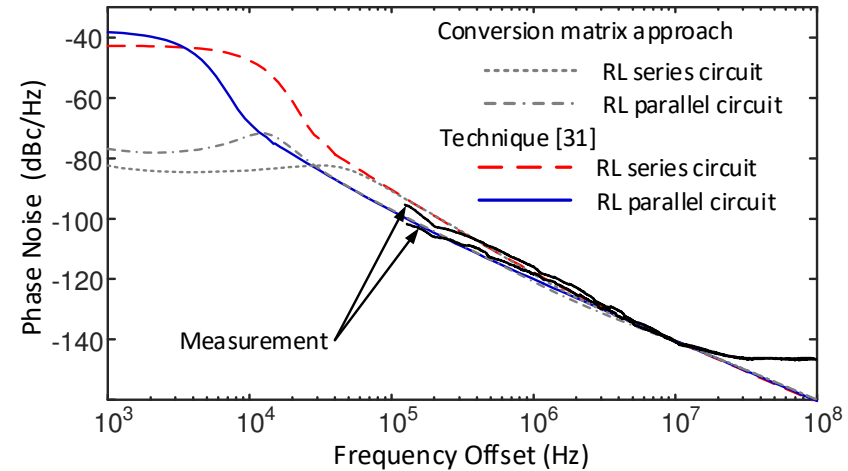

Fig. 13. Comparison of the phase-noise spectra obtained using the conversion-matrix approach and the analysis of the phase variance [31] for two different loads $Z_{s}(f)$ and $Z_{p}(f)$. They correspond to the series R-L and parallel R-L implementations of $\Gamma_{o}=0.4 e^{j 120^{\circ}}$ at $f_{o}=2.598 \mathrm{GHz}$, considered in Section IV.B and Fig. 12. Measurements are superimposed.

\section{CONCLUSION}

A new methodology has been presented for an efficient analysis of the influence of the termination load on the oscillation frequency and output power of autonomous circuits. The methodology requires a low-pass filtering of higher harmonic terms in the output network, so that the termination load may only have an impact at the fundamental frequency. Nevertheless, all the rest of harmonic components, which may be significant at internal nodes, are taken into account in the calculation. The analysis, carried out in harmonic balance, with an arbitrary number of harmonic terms, does not involve any optimization procedures. It should be interesting for the prediction of undesired pulling effects under output mismatch and for an efficient synthesis of oscillator circuits, in order to achieve particular specifications, of oscillation frequency and output power. The analysis is based on the calculation of constant-amplitude contours and constant-frequency contours in the Smith chart. By inspecting these contours, some forms of hysteresis, associated with the variation of termination load at the steady-state oscillation frequency, can be detected. However, in most cases, the stability properties of a given design will depend on the particular implementation of the selected load impedance and its frequency characteristic. The phase-noise spectral density is evaluated through the analysis of the variance of the phase deviation, in combination with the conversion-matrix approach.

\section{REFERENCES}

[1] D. Qiao et al., "Antenna impedance mismatch measurement and correction for adaptive CDMA transceivers," IEEE MTT-S Int. Microwave Symp., Long Beach, CA, USA, Jun. 2005.

[2] A. van Bezooijen, C. Chanlo, and A. van Roermund, "Adaptively preserving power amplifier linearity under antenna mismatch," IEEE MTT-S Int. Microwave Symp., Fort Worth, TX, US, Jun. 2004.

[3] A. Keerti and A. H. Pham, "RF Characterization of SiGe HBT Power Amplifiers Under Load Mismatch", IEEE Trans. Microw. Theory Techn., vol. 55, no. 2, pp. 207-214, Feb. 2007.

[4] W. Karoui and W. T Parra, "A protection circuit for HBT RF Power Amplifier under load mismatch conditions," 6th Int. IEEE Northeast Workshop Circuits Syst. TAISA Conf., Montreal, QC, Jun. 2008, pp. 241-244.

[5] S. Dellier, R. Gourseyrol, J. Collantes, A. Anakabe, G. SoubercazePun, and K. Narendra, "Stability analysis of microwave circuits," IEEE Wireless Microw. Techn. Conf. (WAMICON), Cocoa Beach, FL, USA, Apr. 2012.

[6] K. Narendra, E. Limiti, C. Paoloni, J. M. Collantes, R. Jansen, and S. Yarman, "Vectorially Combined Distributed Power Amplifiers for Software-Defined Radio Applications," IEEE Trans. Microw. Theory Techn., vol. 60, no. 10, pp. 3189-3200, Oct. 2012.

[7] J. F. Imbornone, M. Murphy, R.S. Donahue, and E. Heaney, "New insight into subharmonic oscillation mode of GaAs power amplifiers Under Severe Output Mismatch Condition," IEEE J. Solid-State Circuits, vol. 32, no. 9, pp. 1319-1325, Sep. 1997.

[8] A. Suárez, S. Sancho, M. Pontón, and F. Ramírez, "Advances in the simulation of autonomous microwave circuits," IEEE MTT-S Latin America Microw. Conf., Puerto Vallarta, Mexico, 2016.

[9] A. Suárez, F. Ramírez, and S. Sancho, "Stability Analysis of Power Amplifiers Under Output Mismatch Effects," IEEE Trans. Microw. Theory Techn., vol. 62, no. 10, pp. 2273-2289, Oct. 2014.

[10] A. Suárez, F. Ramírez, and S. Sancho, "Stability criteria for power amplifiers under mismatch effects," IEEE MTT-S Int. Microwave Symp., Phoenix, AZ, USA, May. 2015.

[11] A. Suárez, F. Ramírez, and S. Sancho, "Generalized stability criteria of Power Amplifiers Under Output Mismatch Effects," IEEE Trans. Microw. Theory Techn., vol. 64, no. 10, pp. 2273-2289, Dec. 2015.

[12] A. Anakabe, N. Ayllón, J.M. Collantes, A. Mallet, G. Soubercaze-Pun, and K. Narendra "Automatic pole-zero identification for multivariable large-signal stability analysis of RF and microwave circuits," European Microw. Conf. (EuMC), Paris, France, pp. 477-480, 2010.

[13] J. Jugo, J. Portilla, A. Anakabe, A. Suárez, and J. M. Collantes, "Closed-loop stability analysis of microwave amplifiers," IEEE Electron. Lett., vol. 37, no. 4, pp. 226-228, Feb. 2001.

[14] A. Anakabe et al. "Analysis and elimination of parametric oscillations in monolithic power amplifiers," IEEE MTT-S Int. Microwave Symp. Dig., Seattle, WA, Jun. 2002, pp. 2181-2184.

[15] M. L. Edwards and J. H. Sinsky, "A new criterion for linear 2-port stability using geometrically derived parameters," IEEE Trans. Microw. Theory Techn., vol. 40, no. 12, pp. 2303-2311, Dec. 1992.

[16] J. R. Ford and N. I. Korman, "Stability and frequency pulling of loaded unstabilized oscillators," Proc. IRE, vol. 34, no. 10, pp. 794-799, Oct. 1946. 
[17] U. L. Rohde, A. K. Poddar, and G. Bock, The Design of Modern Microwave Oscillators for Wireless Applications, Wiley, Hoboken, NJ, 2005.

[18] C. Tsironis, "Oscillator Load Pull Measurements Using a Computer Controlled Tuner," 35th ARFTG Conf. Dig., Dallas, TX, USA, 1990, pp. $12-18$.

[19] M. Schott, F. Lenk, and P. Heymann, "On the Load-Pull Effect in MMIC Oscillator Measurements," European Microw. Conf. (EuMC), Munich, Germany, pp. 367-370, 2003.

[20] R. W. Rhea, Discrete Oscillator Design: Linear, Nonlinear, Transient, and Noise Domains, Artech House Publishers, New York, 2010.

[21] J. Obregon and A. P. S. Khanna, "Exact Derivation of the Nonlinear Negative-Resistance Oscillator Pulling Figure," IEEE Trans. Microw. Theory Techn., vol. 30, no. 7, pp. 1109-1111, Jul. 1982.

[22] A. Grebennikov, $R F$ and Microwave Transistor Oscillator Design, Wiley, West Sussex, 2007.

[23] A. Suárez, R. Quéré, M. Camiade and E. Ngoya, "Large signal design of broadband monolithic microwave frequency dividers," IEEE MTT-S Int. Microwave Symp., Albuquerque, NM, USA, 1992, pp. 1595-1598 vol.3.

[24] R. Quéré, E. Ngoya, M. Camiade, A. Suárez, M. Hessane, and J. Obregon, "Large signal design of broadband monolithic microwave frequency dividers and phase-locked oscillators," IEEE Trans. Microw. Theory Techn., vol. 41, no. 11, pp. 1928-1938, Nov. 1993.

[25] U. L. Rohde and A. K. Poddar, "Reconfigurable concurrent oscillators for multi-band multi-mode wireless communication systems," IEEE Sarnoff Symp., Nassau Inn, Princeton, NJ, 2007, pp. 1-6.

[26] U. L. Rohde and A. K. Poddar, "Reconfigurable Concurrent Oscillator (RCO)," IEEE Int. Symp. Personal, Indoor and Mobile Radio Commun., Athens, Greece, 2007, pp. 1-5.

[27] V. Rizzoli and A. Neri, "State of the art and present trends in nonlinear microwave CAD techniques," IEEE Trans. Microw. Theory Techn., vol 36, pp. 343-356, Feb. 1988.

[28] A. Suárez, Analysis and design of autonomous microwave circuits, IEEE-Wiley, Jan. 2009.

[29] B. Razavi, "A Study of phase noise in CMOS oscillators", IEEE J. Solid State Circuits, vol. 31, no. 3, pp. 331-343, 1996.

[30] K. Kundert, "Introduction to RF simulation and its application," Proc. IEEE Bipolar/BiCMOS Circuits Techn. Meeting, vol. 34, no. 9, pp. 67 78, Sep. 1998.

[31] S. Sancho, A. Suarez, and F. Ramirez, "General Phase-Noise Analysis from the Variance of the Phase Deviation," IEEE Trans. Microw. Theory Techn., vol. 61, no. 1, pp. 472-481, Jun. 2013.

[32] V. Rizzoli, F. Mastri, and D. Masotti, "General noise analysis of nonlinear microwave circuits by the piecewise harmonic balance technique," IEEE Trans. Microw. Theory Techn., vol. 42, no. 5, pp. 807-819, May. 1994

[33] J. M. Paillot, J. C. Nallatamby, M. Hessane, R. Quéré, M. Prigent, and J. Rousset, "A general program for steady state, stability, and FM noise analysis of microwave oscillators," IEEE MTT-S Int. Microwave Symp., Dallas, Texas, USA, 1990, pp. 1287-1290.

[34] J. M. Rollett, "Stability and power-gain invariants of linear two ports," Ins. Radio Engineers Trans, on Circuit Theory, vol. 9, no. 1, pp. 29-32, Mar. 1962.

[35] F. Ramírez, E. de Cos, and A. Suárez, "Nonlinear analysis Tools for the Optimized Design of Harmonic-Injection Dividers," IEEE Trans. Microw. Theory Techn., vol. 51, no. 6, pp. 1752-1762, Jun. 2003.

[36] K. Kurokawa, "Noise in synchronized oscillators," IEEE Trans. Microw. Theory Techn., vol. 16, no. 4, pp. 234-240, Apr. 1968.

[37] J. Guckenheimer and P. Holmes, Nonlinear oscillations, dynamical systems and bifurcations of vector fields, New York: Springer-Verlag, 1990.

[38] S. Wiggins, Introduction to Applied Nonlinear Dynamical Systems and Chaos, New York: Springer-Verlag, 1990.

[39] A. Suárez, E. Fernández, F. Ramírez, and S. Sancho, "Stability and Bifurcation Analysis of Self-Oscillating Quasi-Periodic Regimes," IEEE Trans. on Microwave Theory and Techn., vol. 60, no. 3, pp. 528-541, Mar. 2012.

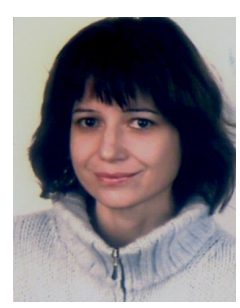

Almudena Suárez (M'96-SM'01-F'12) was born in Santander, Spain. She received the Electronic Physics and Ph.D. degrees from the University of Cantabria, Santander, Spain, in 1987 and 1992, respectively, and the Ph.D. degree in electronics from the University of Limoges, Limoges, France, in 1993. She is currently a Full Professor with the Communications Engineering Department, University of Cantabria. She co-authored Stability Analysis of Nonlinear Microwave Circuits (Artech House, 2003) and authored Analysis and Design of Autonomous Microwave Circuits (IEEE-Wiley, 2009). Prof. Suárez is a member of the Technical Committees of the IEEE International Microwave Symposium (IMS), the European Microwave Conference. She was an IEEE Distinguished Microwave Lecturer from 2006 to 2008 , with the talk "Stability analysis and stabilization of power amplifiers." She is the editor-in-chief of International Journal of Microwave and Wireless Technologies, from Cambridge Journals. She is an associate editor of IEEE Microwave Magazine. She is a member of the "Board of Directors" of the European Microwave Association. She was the coordinator of the Communications and Electronic Technology Area for the Spanish National Evaluation and Foresight Agency (ANEP) between 2009 and 2013.

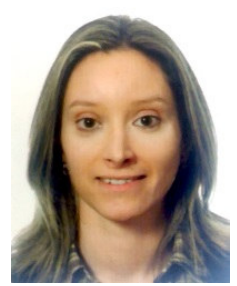

Mabel Pontón (S'08-M'11) was born in Santander, Spain. She received the bachelor's degree in telecommunication engineering, master's degree in information technologies and wireless communications systems, and Ph.D. degree from the University of Cantabria, Santander, in 2004, 2008, and 2010, respectively.

In 2006, she joined the Communications Engineering Department, University of Cantabria. From 2011 to 2013, she was with the Group of Electronic Design and Applications, Georgia Institute of Technology, Atlanta, GA, USA, as a Post-Doctoral Research Fellow.

Her current research interests include the nonlinear analysis and simulation of radiofrequency and microwave circuits, with an emphasis on phase-noise, stability, and bifurcation analysis of complex oscillator topologies.

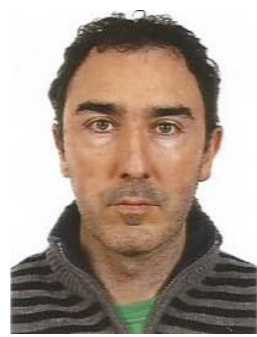

Sergio Sancho received the degree in Physics from Basque Country University in 1997. In 1998 he joined the Communications Engineering Department of the University of Cantabria, Spain, where he received the $\mathrm{Ph} . \mathrm{D}$. degree in Electronic Engineering in February 2002. At present, he works at the University of Cantabria, as an Associate Professor of its Communications Engineering Department. His research interests include the nonlinear analysis of microwave autonomous circuits and frequency synthesizers, including stochastic and phase-noise analysis.

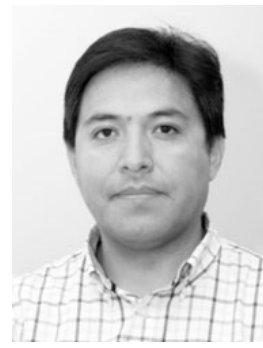

Franco Ramírez (S'03-A'04-M'05-SM'16) was born in Potosí, Bolivia. He obtained a degree in electronic systems engineering degree from the Military School of Engineering (EMI) in La Paz, Bolivia, in 2000 and the Ph.D. degree in communications engineering from the University of Cantabria, Santander, Spain in 2005. From 1999 to 2000 he worked for Ericsson de Bolivia Telecomunicaciones, where he was involved in several projects related with GSM and TDMA technologies. At present he is an Associate Professor at the Communications Engineering Department of the University of Cantabria. His research interests include phase noise, stability and the development of nonlinear techniques for the analysis and design of autonomous microwave circuits. 